\title{
Validation of a Serum Analysis Method to Analyze Antihistamines by Capillary Electrophoresis
}

\author{
J. Peris-Vicente, S. Carda-Broch, and J. Esteve-Romero \\ Departament de Química Física i Analítica, ESTCE, Universitat Jaume I, Campus Riu Sec, 12071 Castellón de la Plana, Spain \\ Correspondence should be addressed to J. Peris-Vicente; vicentej@uji.es
}

Received 14 June 2014; Accepted 21 July 2014; Published 25 August 2014

Academic Editor: Junsheng Yu

Copyright ( 2014 J. Peris-Vicente et al. This is an open access article distributed under the Creative Commons Attribution License, which permits unrestricted use, distribution, and reproduction in any medium, provided the original work is properly cited.

\begin{abstract}
The validation of an electrophoresis-based analytical method to quantify 17 antihistamines in pharmaceutical formulations and serum is described. Then, whether the methodology provides true values with low uncertainty and is able to detect the concentration range level of analyte usually found in the matrix was evaluated. The analytical method was validated following the recommendations of an official guide to provide more reliability to the results. The ICH Harmonized Tripartite Guideline was selected because it was especially developed for analysis of drugs. The guide and the following required validation parameters, selectivity, calibration range, linearity, limit of detection, limit of quantification, inter- and intraday accuracy and precision, and robustness, were described. The method was inexpensive, fast, simple, environmentally friendly, and useful for routine analysis. The methodology was successfully validated and applied to commercial pharmaceutical formulations and spiked blank serum samples.
\end{abstract}

\section{Introduction}

Antihistamines are widely used to provide relief of allergic symptoms caused by histamine release. They are administered as decongestants for the treatment of coughs, colds, and allergies [1]. Structurally, antihistamines are substituted ethylamines $[2,3]$. The structure can be seen in Figure 1. In order to prescribe the adequate dose of antihistamines, the accurate amount of analytes in pharmaceutical formulations has to be known. Moreover, the measurement of the concentration of antihistamines in blood is useful to study the way of metabolization of the compounds. Therefore, the development of an analytical methodology to quantify antihistamines in pharmaceutical formulations is of the utmost importance to study their effects on the human body [4].

Recently, a capillary electrophoresis method has been proposed to detect antihistamines in pharmaceuticals and blood [3]. This method proposes an easy sample preparation: the solubilization of pharmaceutical formulations and serum in a micellar solution. The main electrophoresis conditions (capillary length, $\mathrm{pH}$ buffer, buffer concentration, injection pressure, applied voltage, and UV) were optimized. This method show important advantages, as low cost, high separation efficiency, short analysis times, low operational costs, and fast method development [3].
Once the methodology was developed, it was validated to prove that it was suitable for its intended purpose: the reliable quantification of antihistamines in pharmaceuticals and blood with adequate sensitivity and the possibility to apply it in routine analysis. The validation assesses the quality of the results provided by the method. It is also helpful to establish the ability of the staff and the quality of laboratory instrumentation. The validation requires to study the variability of the signal (which should be as low as possible) to evaluate the closeness between the provided and true value and the determination of the interval of applicability (which should include the permitted levels). The procedures and scope of validation are not always the same and must be established depending on the scope of the analysis, the analyte, and the matrix. The validation is performed by measuring several statistical parameters, as selectivity, sensitivity, limit of detection, limit of quantification, accuracy, precision, and robustness. The measurement of the concentration of analyte using a validated analytical method provides reliability to the results and is sometimes required in scientific journals or industry reports. A method must be validated to be used for clinical purposes, as clinicians take important decisions about the health of the patient, as well as the prescription of pharmaceutical formulations (dose and frequency) $[5,6]$. 
(1) Ethylenediamine derivatives<smiles>CN(C)CCCN(Cc1ccc(O)cc1)c1ccccn1</smiles>

Pyrilamine (1)

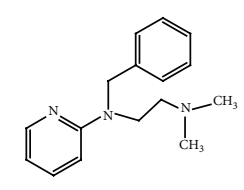

Tripelennamine (2)

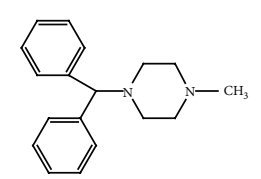

Cyclizine (11)

(4) Piperazine derivatives

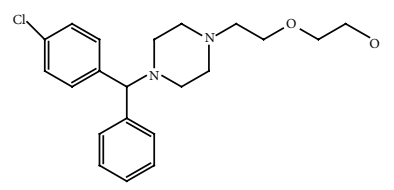

Hydroxyzine (12)

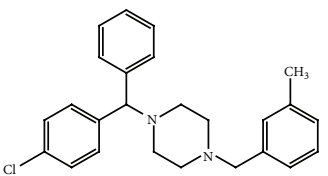

Meclizine (13)

(2) Ethanolamine derivatives

(5) Other derivatives

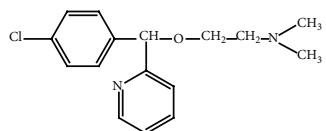

Carbinoxamine (3)

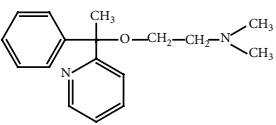

Doxylamine (5)

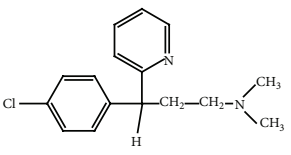

Chlorpheniramine (7)

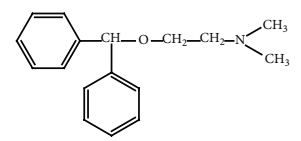

Diphenhydramine (4)

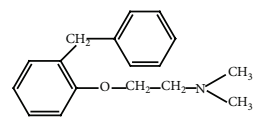

Phenyltoloxamine (6)

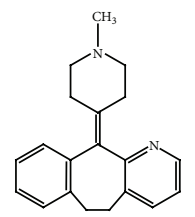

Azatadine (14)<smiles>CN1CCC(=C2c3ccccc3CCc3ccccc32)CC1</smiles>

Cyproheptadine (15)

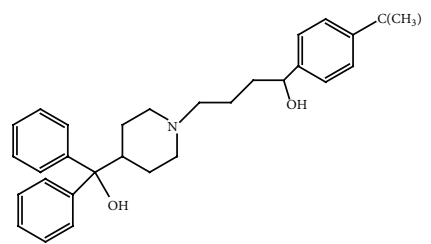

Terfenadine (17)

(3) Propylamine derivatives

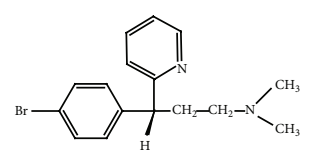

Dexbrompheniramine (8)

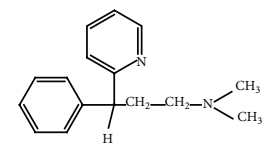

Pheniramine (9)

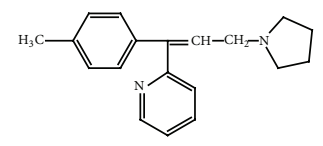

Tripolidine (10)

Figure 1: Structure of the antihistamines.

Method validation is considered to be very closely related to method development. Many of the method performance parameters that are associated with method validation are in fact usually evaluated, as part of method development. Currently, there is a wide range of recognized international organizations which have developed guidelines on validation (AOAC, FAO, FDA, ICH, WHO, IUPAC, USP, and EURACHEM, among others). Each one suggested the calculation of several validation parameters. The existence of many protocols and guidelines can confuse the analysts in their selection, and this can finally avoid the validation of a method. The selection of a guideline is made on the basis of the analyte, the sample, the scope of the analysis, and the geographic zone of application [5-10].

The aim of the paper is to thoroughly discuss the validation protocol of the analytical procedure developed in [3]. This method was developed to quantify the antihistamines indicated in Figure 1 in pharmaceutical formulations and serum by CE-UV and successfully validated following the requirements of the International Conference on Harmonisation of Technical Requirements for Registration of Pharmaceuticals for Human Use (ICH Harmonization Tripartite Guideline). The validation parameters required by the guide
[8] and obtained results in [3] were described as follows: specificity, calibration range, linearity, sensitivity, limit of detection, limit of quantification, accuracy, precision, and robustness. According to the excellent results obtained in the validation, the suitability of the method for routine analysis of real samples was proven.

\section{Description of the Validation Guide}

The "ICH Harmonization Tripartite Guideline: Validation of Analytical Procedures: Text and Methodology Q2(R1)" has been developed by the ICH Expert Working Group of the International Conference on Harmonization of Technical Requirements for Registration of Pharmaceuticals for Human Use in October 27, 1994, and the last modification was performed in November 2005. It has been subject to consultation by the regulatory parties, in accordance with the ICH validation process [8].

This guideline presents a discussion of the parameters for consideration during the validation of the analytical procedures of drugs and pharmaceuticals in all kinds of matrices included as part of registration application submitted within the EC, Japan, and USA. The document does not necessarily 
seek to cover the testing that may be required for registration in, or export to, other areas of the world. Furthermore, this text presentation serves as a collection of terms, as well as their definitions, and is not intended to provide direction on how to accomplish validation. These terms and definitions are meant to bridge the differences that often exist between various compendia and regulators of the EC, Japan, and USA. Once validated, the method will remain "validated" when applied in the same laboratory and using the same experimental procedure and electrophoresis conditions. If one condition is changed or the methodology is applied in another laboratory, it must be validated $[3,8]$.

The ICH Harmonized Tripartite Guideline was chosen for the validation of the analytical methodology to quantify antihistamines in pharmaceutical formulations and serum because it is specifically addressed to drugs and pharmaceuticals $[3,8]$. The ICH Harmonized Tripartite Guideline requires the measurements of several statistical parameters and obtains values in agreement with those required by the guideline. The validation can be performed using spiked samples or using standard solutions in water, even if the first option is preferable. The main validation parameters and requirements are described as follows $[3,5,8]$.

2.1. Specificity. Specificity is the capacity to unequivocally identify the analyte in the presence of components which may be expected to be in the sample. In capillary electrophoresis, it means that there is no other compound with the migration time similar to the analyte. The specificity should be tested analyzing the studied analytes spiked in the blank matrices (it cannot be studied using standard solutions in water). A solution of the analyte at LOQ level with potential interfering substances (endogenous compounds and other drugs possibly prescribed together, analyte metabolites, and decomposition products) should be analyzed, and they must appear enough far from the analyte, so that they do not interfere in the measuring of the area of the peak of analyte.

2.2. Calibration Curve and Linearity. The calibration curve is represented by a first-grade equation relating the signal provided by the detector and the concentration. The calibration curve is constructed using the least-square linear regression by plotting the area of the electrophoresis peak corresponding to the analyte versus the concentration, using a minimum of five increasing values. The general form is $S=\left(A \pm s_{A}\right)$ [concentration] $+\left(B \pm s_{B}\right)$, where $S$ is the detector signal, $A$ is the slope, and $B$ is the $y$-intercept, both with the corresponding error. The calibration curve can be constructed using standard solutions or spiked samples. This last option is preferred because the matrix effects would be already included in the linear parameters.

The linearity is the ability within a given range to obtain test results which are directly proportional to the concentration of analyte in the sample with suitable accuracy and precision. Linearity is evaluated by the coefficient of determination $\left(r^{2}\right)$, obtained with the calibration curve by least-square linear regression.
A $r^{2}$ close to 1 indicates adequate linearity, whereas a $r^{2}$ close to 0 indicates the total absence of proportionality. The ideally $y$-intercept is close to 0 ; a different value indicates a bias in measures. LOQ must be inside the calibration curve and zero level never can be included. The regression coefficient should be over 0.99 .

2.3. Linear Range. Calibration range is the minimal and maximal concentration values at which the calibration curve is applicable. The required calibration range depends on the expected concentration in the samples. In the case of a drug substance or a finished drug, the linear range must cover from 80 to $120 \%$ of the test concentration; for content uniformity: a minimum of 70 to 130 percent of the test concentration; for dissolution testing: $\pm 20 \%$ over the specified range; a pharmaceutical in a formulation: from 0 to $110 \%$ to the label claim and an impurity from 0 to $120 \%$.

2.4. Sensitivity. The sensitivity is the capacity of the analytical methodology to distinguish small differences in the concentration of the analyte. It is evaluated through the slope of the calibration curve.

2.5. Limit of Detection. Limit of detection (LOD) is the minimal concentration of an analyte that the analytical method can reliably differentiate from background noise. However, at this level, the analyte cannot be quantified with suitable accuracy and precision; thus it is normally under the lower level of the calibration curve. It is calculated by visual appreciation, as the concentration which provides a signal-tonoise ratio over 3 , or statistically as three times the standard deviation of a blank sample $(n=10)$ or standard deviation of $y$-intercept of a calibration curve at concentrations near LOD. As the signal noise depends on endogenous compounds in the matrix, LOD must be determined using analyte-free or spiked samples. The limit of detection should be under the minimal amount of the studied compound expected to be present in a real sample.

2.6. Limit of Quantification. Limit of quantification (LOQ) is the minimal amount of analyte which can be quantified with reliability (adequate accuracy and precision). The limit of quantification can be determined by visual evaluation, as the concentration providing a signal-to-noise ratio over 10 , or calculated as ten times the standard deviation of a blank, standard deviation of the blank, or the standard deviation of $y$-intercept of a calibration curve taking concentrations near LOQ, divided by the sensitivity. The limit of quantification should be under the minimal amount of analyte which can be normally found in a sample and can be in the linear range. As for LOD, LOQ should be measured in matrix sample.

2.7. Accuracy. The accuracy is defined as closeness between the concentration of the analyte in a standard solution or in a spiked sample, obtained through the proposed calibration curve, and the true value. The accuracy is expressed as bias $(\%)=([$ calculated concentration $]-$ [true concentration] $) /[$ true concentration] $\times 100$; then a bias near 0 means 
excellent accuracy. To evaluate the bias, the measurements accuracy is determined by several replicates as follows.

(a) Intraday measurement: replicates were taken in the same day.

(b) Interday measurement: average of several measurements of the intraday values was taken in the same laboratory at different days over a period of several months. The influence of other sources of variability (analyst, instrumentation, reagent supplier, etc.) can be studied in the same way, by calculating the accuracy of several measures taken by changing the studied source of variance (different analysts, or using a different instrument, or using reagents purchased from several suppliers, etc.).

(c) Interlaboratory: average of several measurements of the interday values was taken by different laboratories, but applying the same analytical procedure and electrophoresis conditions.

Accuracy should be evaluated at a minimum of nine determinations, for instance, three replicates of three concentrations. The solutions must be different from those prepared for the calibration.

2.8. Precision. The precision is considered as the concordance of the signals obtained by several replicates of the same analyte solution. It is calculated as the relative standard deviation (R.S.D., \%) of the peak area obtained from several analyses of multiple aliquots of a single homogeneous volume of matrix; then a R.S.D. close to $0 \%$ indicates an exceptional precision. Precision should be evaluated by nine measurements, normally, at three levels a minimum of three replicates. Normally, the same experiments are used to determine accuracy and precision.

The precision was intraday, interday (R.S.D. of the average of diverse measurements performed in several days), and interlaboratory (R.S.D. of the average of the measurements taken in each laboratory) determined. The resulting parameters are also named repeatability, intermediate precision, and reproducibility, respectively. As in accuracy, the influence of other method parameters, as analyst, instrumentation, or supplier reagents, can also be evaluated as intermediate precision.

2.9. Recovery and Label Claim. The recovery is the closeness between the concentration obtained applying the analytical method to a spiked sample and the true concentration spiked to the matrix. The recovery is defined as the ratio calculated concentration/spiked concentration; then the more outstanding value is $100 \%$. The recovery should be taken at several concentration levels. Label claim is the recovery percentage by determining the amount of antihistamine in a pharmaceutical formulation.

If the calibration has been performed using standard solutions, the recovery has to be mandatory performed using spiked blank matrix. If the calibration has been carried out in the studied matrix, but the analyte is expected to be found in other matrices, the recovery has to be repeated in these new matrices.
2.10. Robustness. The robustness of an analytical method is the ability to remain unaltered by slight and premeditated variation in experimental conditions and provides an indication of its stability during normal use. The aim is to assess if the normal oscillation of experimental parameters will enhance the uncertainty of the measures of migration time and peak area.

The migration time and peak area of the analyte are measured analyzing a medium concentration level (a minimum of three replicates) at three levels of each electrophoresis parameter (maintaining the others constant): the optimal value; slightly under (10\%) the optimal value and slightly over (10\%) the optimal value. The variation of migration time and peak area was determined by calculating the average value, standard deviation, and R.S.D. of the obtained values.

The influence of the main electrophoresis parameters should be tested: buffer $\mathrm{pH}$, phosphate buffer concentration, applied voltage, and injection pressure.

\section{Analysis of Antihistamines in Pharmaceuticals and Serum by Capillary Electrophoresis}

An analytical methodology to simultaneously quantify 17 antihistamines in pharmaceuticals and serum was optimized and validated following the ICH Harmonized Tripartite Guideline [3].

\subsection{Experimental Procedure and Chromatographic Conditions.} The experimental procedure was quite, fast, and simple: the pharmaceutical formulations (capsules, powder, and lozenge) and serum samples (previously centrifuged) were 1/10-diluted in $0.15 \mathrm{M} / \mathrm{SDS}-0.9 \% \mathrm{NaCl}$, filtered, and injected. The main electrophoresis conditions $(\mathrm{pH}$, applied voltage, organic modifier, and injection pressure) were selected on the basis on maximal resolution at minimum analysis time and adequate shape of the peaks. A standard solution containing the studied antihistamines was analyzed varying the electrophoresis conditions to find the optimal values. The separation was finally performed by applying the following conditions: capillary effective length, $24 \mathrm{~cm}$; injection pressure, $2 \mathrm{psi} \cdot \mathrm{s}^{-1}$; phosphate buffer, $20 \mathrm{mM}$ and $\mathrm{pH} 2.0$; UV detection at $214 \mathrm{~nm}$; and applied voltage, $5 \mathrm{kV}$. Under these conditions, the studied antihistamines were separated in less than $10 \mathrm{~min}$ [3].

3.2. Method Validation. Pharmaceutical formulation matrix is difficult to imitate, as there is no "pharmaceutical formulation" without active ingredient. However, as formulations are dissolved in SDS/ $\mathrm{NaCl}$ solution, it can be considered that a standard solution diluted in $\mathrm{SDS} / \mathrm{NaCl}$ imitates the pharmaceutical formulation samples. Therefore, the validation was carried out in SDS/ $\mathrm{NaCl}$ solution and in spiked serum samples. As the results were similar in both cases, those corresponding to serum were discussed [3].

In order to test the specificity, a solution was prepared by dissolving antihistamines and the most common compounds, as paracetamol, caffeine, and saccharose, at a similar concentration to that found as excipients in pharmaceuticals 
with a view to creating a similar real matrix. The specificity was tested by analyzing (10 replicates) this solution directly diluted in SDS solution and spiked in a blank serum sample. The studied antihistamines were separately analyzed in five groups depending on the structure and the migration time (min) including (a) ethylenediamine derivatives (tripelennamine, 3.3, and pyrilamine, 3.5); (b) ethanolamine derivatives (carbinoxamine, 5.6; doxylamine, 5.7; diphenhydramine, 8.9; and phenyltoloxamine, 9.5); (c) propylamine derivatives (triprolidine, 4.8; pheniramine, 4.9; dexbrompheniramine, 5.2; and chlorpheniramine, 5.3); (d) piperazine derivatives (cyclizine, 4.9; hydroxyzine, 6.0; and meclizine, 6.5); and (e) other derivatives (azatadine, 5.2; cyproheptadine, 6.5; loratadine, 7.2; and terfenadine, 8.3). The electropherograms obtained by the analysis of the five studied groups of antihistamines (17 analytes) can be seen in [3]. Even if the migration time of some of the antihistamines belonging to the same group was close, there was no overlapping because of the high efficiency of capillary electrophoresis [3]. Moreover, no interference between the antihistamines and endogenous compounds in serum was observed, and then it can be assessed that the peak area of the studied antihistamines can be reliably measured.

The calibration was simultaneously performed for all studied antihistamines. The calibration curves were constructed using several samples at increasing concentrations from 0.5 to $20 \mu \mathrm{g} / \mathrm{mL}$ of each antihistamine (6 replicates). The calibration was repeated five times over a 2 -month period and the average values of slope, $y$-intercept, and determination coefficient. For each studied drug, an adequate linearity was found in the studied range $\left(r^{2}>0.9991\right)$. LOD and LOQ were calculated as three and ten times the standard deviation of the low level of calibration $(1 \mu \mathrm{g} / \mathrm{mL})$, measured by 10 replicates, divided by the slope of the calibration curve. Limit of detection and limit of quantification were 2-20 and 9$61 \mathrm{ppb}$, respectively. These values are under those found in serum of patients who take antihistamines.

The accuracy and precision of the methodology were evaluated using blank serum sample spiked at three concentrations. Several values should be tested to cover several ranges of concentration because accuracy and precision depend on the amount of analyte. In fact, the values at low concentrations are supposed to be more uncertain and less accurate than at higher ones. The selected concentrations were LOQ and two medium concentrations ( 2.5 and $5 \mu \mathrm{g} / \mathrm{mL}$ ). Intra- and interday precision were $<2.1$ and $<3.0 \%$, whereas intra- and interday accuracy were between 97.5$100.5 \%$ and $96.5-101.5 \%$, respectively. Interlaboratory precision was not evaluated. These results are excellent and indicated that the method is able to provide concentration of the antihistamines close to the true value with low uncertainty in calibration range.

The robustness was evaluated using a blank serum sample spiked with $1 \mu \mathrm{g} / \mathrm{mL}$ of all the studied antihistamines. The tested electrophoresis parameters were buffer $\mathrm{pH}$ (2.9-3.1), buffer phosphate concentration (19-21 mM), applied voltage $(4.5-5.5 \mathrm{kV})$, and injection pressure $\left(1.9-2.1 \mathrm{psi} \cdot \mathrm{s}^{-1}\right)$. Each level was taken as the average of 6 measurements. The variation of migration time was calculated for each antihistamine and was R.S.D. $<5.6 \%$. Therefore, slight oscillations which can be produced during the normal handling during the experimental procedure do significantly affect the migration time.

According to the results obtained for validation, it can be considered that applying the method, a similar analyte concentration provides a similar response in the detector; the measured concentrations of the 17 studied antihistamines are close to the true value in a range of concentrations at which the analytes can be found in pharmaceutical formulations and in serum.

The applicability of the method to real samples was studied by analyzing several pharmaceutical formulations and spiked serum samples with a known concentration of antihistamines. Label claims were $95.7-103.4 \%$ with R.S.D. $<4 \%$, whereas recoveries were $97.1-101.9 \%$ with R.S.D. $<3.6 \%$. These results for validation indicate that the method can be used in routine analysis to check the amount of active principle in pharmaceutical formulation and to measure the level of antihistamine in serum.

The use of micellar solutions as sample solvent allows the solubilization without extraction, and then the experimental procedure is strongly expedited. Moreover, the method was able to separate the 17 antihistamines (distributed in 5 groups depending on the structure) in only $12 \mathrm{~min}$. Together to the utilization of an autosampler injector, a large amount of real samples can be analyzed in a reduced time with a minimal manipulation by an operator. On the other hand, considering the reduced volume of organic solvent, due to the use of micellar solutions and in the buffer, and the low volume injected into the electrophoresis system, the method can be considered environment-friendly.

\section{Conclusions}

The validation has been proven as an essential step in the development of a method to analyze antihistamines in pharmaceutical formulations and serum by electrophoresis. The ICH Harmonized Tripartite Guideline (especially devoted to drugs) provides the useful information to correctly design and achieve the validation and supply more reliability to the methodology. According to its requirements, the validation is performed after the optimization of experimental parameters in the same matrix of the real samples $(\mathrm{SDS} / \mathrm{NaCl}$ solution and serum), in order to include the possible matrix effects in the results. The validation parameters calculated were calibration range, linearity, limit of detection, limit of quantification, inter- and intraday accuracy and precision, robustness, and recovery. As the values are inside the recommendations of the guideline, the method was considered as validated. Therefore, the suitability of the method has been established: the amount usually found in the sample can be detected, the signals were taken with low uncertainty, and the calculated concentrations in the sample are close to the true value. The ability of the laboratory staff has also been evaluated.

\section{Conflict of Interests}

The authors declare that they have no financial/commercial conflict of interests. 


\section{Acknowledgment}

The work was supported by the Project P1.1B2012-36 funded by the Universitat Jaume I.

\section{References}

[1] J. E. F. Reynolds, Martindale: The Extra Pharmacopoeia, The Pharmaceutical Press, London, UK, 30th edition, 1993.

[2] A. C. Moffat, M. D. Osselton, W. Brian, and J. Watts, Clarkes Analysis of Drugs and Poisons, Pharmaceutical Press, London, UK, 2004.

[3] M. Rambla-Alegre, J. Peris-Vicente, J. Esteve-Romero, M. Capella-Peiró, and D. Bose, "Capillary electrophoresis determination of antihistamines in serum and pharmaceuticals," Analytica Chimica Acta, vol. 666, no. 1-2, pp. 102-109, 2010.

[4] H. Fernández, F. J. Rupérez, and C. Barbas, "Capillary electrophoresis determination of loratadine and related impurities," Journal of Pharmaceutical and Biomedical Analysis, vol. 31, no. 3, pp. 499-506, 2003.

[5] S. Kollipara, G. Bende, N. Agarwal, B. Varshney, and J. Paliwal, "International guidelines for bioanalytical method validation: a comparison and discussion on current scenario," Chromatographia, vol. 73, no. 3-4, pp. 201-217, 2011.

[6] J. Peris-Vicente, S. Carda-Broch, J. Esteve-Romero et al., "Validation of micellar LC-based methods applied to analyze foodstuffs," Bioanalysis, vol. 5, no. 4, pp. 481-494, 2013.

[7] Food and Drug Administration, Guidance for Industry: Bioanalytical Method Validation, Food and Drug Administration, Rockville, Md, USA, 2001, http://www.fda.gov/downloads/ Drugs/Guidances/ucm070107.pdf.

[8] ICH Harmonized Tripartite Guideline, Validation of Analytical Procedures: Text and Methodologies Q2(R1), ICH, Geneva, Switzerland, http://www.ich.org/fileadmin/Public_Web_Site/ ICH_Products/Guidelines/Quality/Q2_R1/Step4/Q2_R1_Guideline.pdf.

[9] Guidance for Industry PAT-A Framework for Innovative Pharmaceutical Development, Manufacturing, and Quality Assurance, Food and Drug Administration, Rockville, Md, USA, 2004, http://www.fda.gov/downloads/Drugs/Guidances/ ucm070305.pdf.

[10] Commission Decision 2002/657/EC 2002, "Implementing Council Directive 96/23/EC concerning the performance of analytical methods and the interpretation of results," Official Journal of the European Communities, vol. L221, pp. 8-36, 2002. 

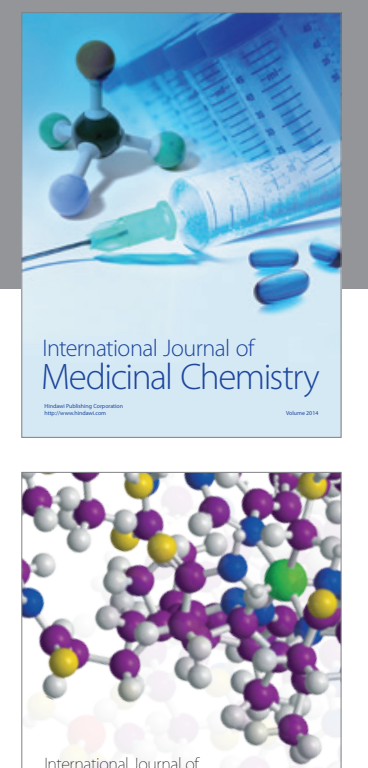

\section{Carbohydrate} Chemistry

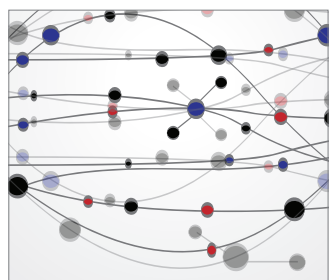

The Scientific World Journal
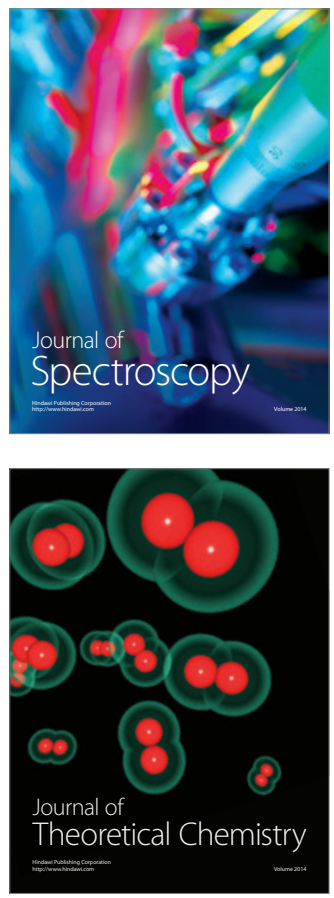
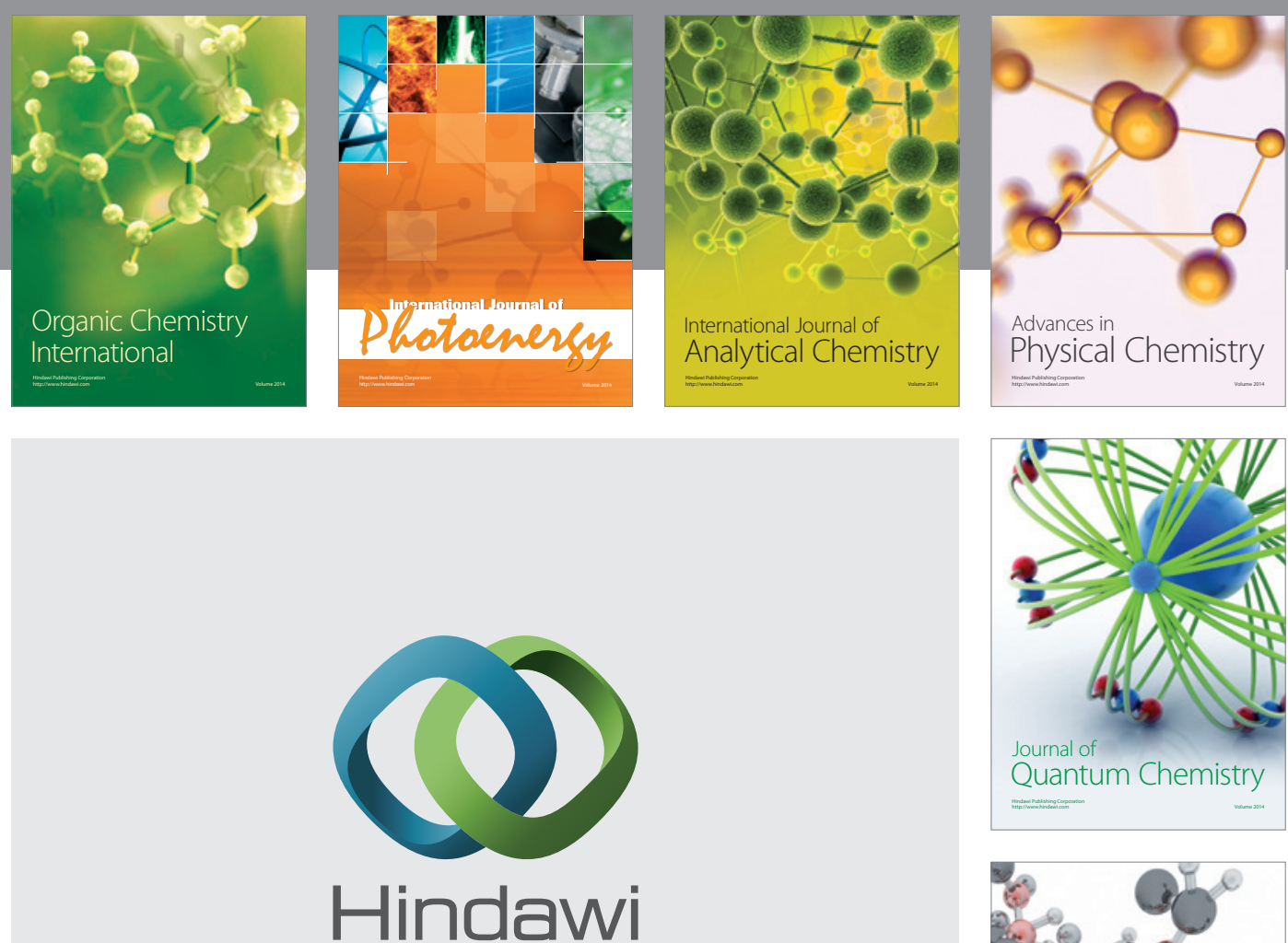

Submit your manuscripts at

http://www.hindawi.com

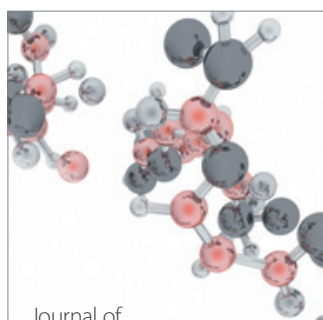

Analytical Methods

in Chemistry

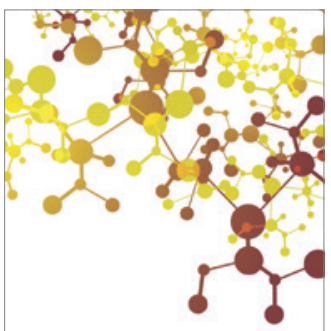

Journal of

Applied Chemistry

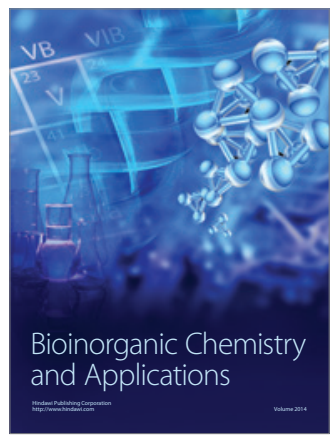

Inorganic Chemistry
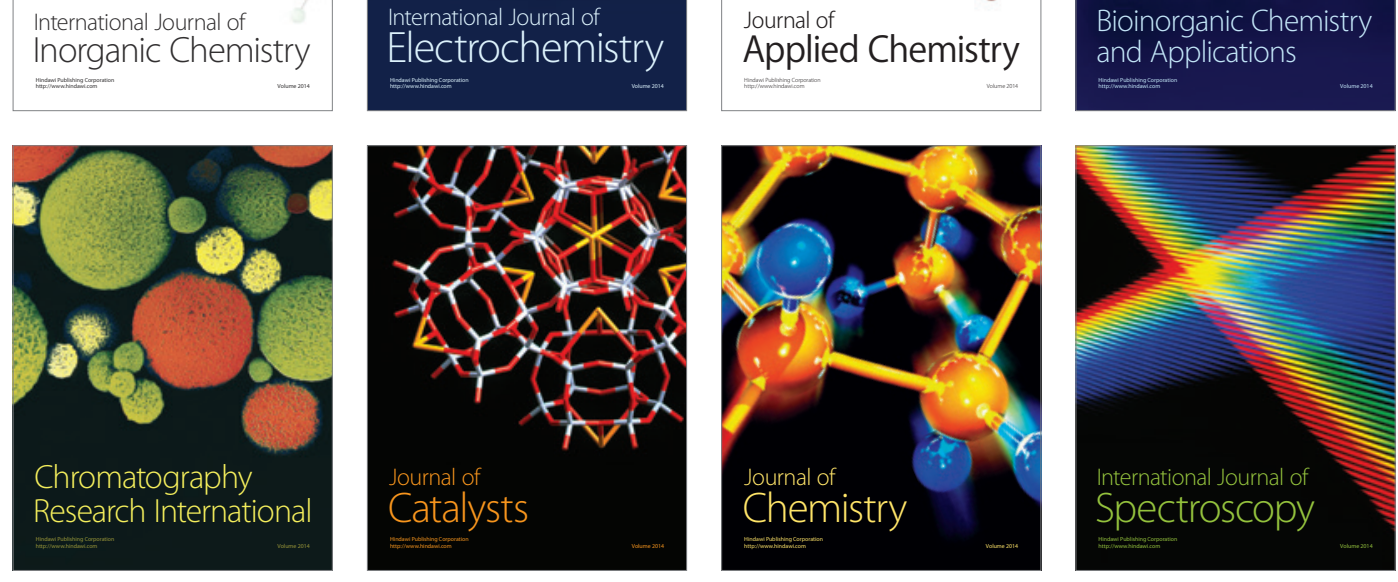